\title{
Structure atomique interfaciale et atténuation du courant supraconducteur par les joints de grains dans les céramiques $\mathrm{YBa}_{2} \mathrm{Cu}_{3} \mathrm{O}_{7-x}$
}

\author{
J. Y. Laval, M. Drouet et W. Swiatnicki \\ Laboratoire de Physique du Solide, CNRS-ESPCI, 10 rue Vauquelın, 75231 Paris Cedex 05, \\ France
}

(Reçu le 31 mars 1994, révisé le 5 mai 1994, accepté le 9 mai 1994)

\begin{abstract}
Résumé. - Nous avons tout d'abord répertorié les différents types de joınts présents dans une céramique polycristalline frıttée. On distingue deux types de joints : les plus fréquents sont ceux contenant le plan $(a b)$. L'exemple le plus caractéristıque est donné par le joint $\Sigma_{\mathrm{Q}} 3$ $\left(c_{1} \perp c_{2}\right)$ qui présente une structure atomique remarquablement continue. Le deuxıème type de joints ne contient pas le plan $(a b)$. Nous montrons que leur structure atomique interfaciale n'est perturbée que sur quelques plans atomiques. Elle devient presque parfaite lorsque les grans adjacents présentent une relatıon de concidence. Parallèlement, nous avons effectué des mesures locales d'atténuation du courant critıque sur ces différents types de joints. Nous montrons que seuls certains joints de coincidence ne contenant pas le plan $(a b)$ atténuent faiblement le courant critique.

Abstract. - Two types of boundaries can be observed in polycrystalline ceramics. The most frequent type hold the $(a b)$ plane. The most strikıng example is given by the coincidence $\Sigma_{0} 3$ boundary $(c, \perp c$,$) whıch exhibits a highly continuous atomic structure. The second type do$ not hold the $(a b)$ plane. It is shown that its interfacial atomic structure is disturbed on a few atomic planes only. Furthermore this structure becomes nearly perfect when abutting grains present a coincidence relatıonshıp. In parallel, local critical current measurements have been carried out on these different types of boundaries. It is shown that only coincidence boundaries which do not hold the $(a b)$ plane display a weak attenuation of the supercurrent.
\end{abstract}

\section{Introduction.}

Nous avons montré précédemment, sur des céramiques $\mathrm{YBa}_{2} \mathrm{Cu}_{3} \mathrm{O}_{7-}$, texturées, que les joints transverses, par rapport à la texture, présentaient de faibles barrières électriques à l'ambiante, contrairement aux interfaces longitudinales [1]. De plus nous avons pu établir une relation entre le comportement électrique du joint et son énergie. Ainsi les joints transverses se caractérisent par l'absence de phase intergranulaire et par une géométrie particulière (plan de 
joint de faible indice et/ou relation d'orientation entre les grains). Cette relation explique bien pourquoi les courants critiques de ces matériaux sont élevés : le courant supraconducteur se propage parallèlement aux interfaces longitudinales et n'est que faiblement atténué à la traversée des joints transverses.

Nous avons étendu cette étude au cas général des céramiques polycristallines frittées, afin de recenser des types de joints favorables, parmi l'ensemble des joints de grains de ce matériau [2]. De plus, afin de tester le comportement supraconducteur de ces joints, nous avons développé des mesures locales du courant critique sur des bicristaux massifs représentatifs des différents types de joints.

Compte tenu de la très faible longueur de cohérence des céramiques $\mathrm{YBa}_{2} \mathrm{Cu}_{3} \mathrm{O}_{7-\text {, }}$ $(0,3<\xi<1,5 \mathrm{~nm})$, la compréhension du comportement électrique des joints implique de connaître leur structure jusqu'à l'échelle atomique. Aussi nous sommes nous proposés de relier directement l'atténuation du courant critique à la cristallographie et à la structure atomique des joints de grains dans les céramiques $\mathrm{YBa}_{2} \mathrm{Cu}_{3} \mathrm{O}_{7-1}$ frittées.

\section{Méthodes expérimentales.}

MATÉRIAUX. - Trois types de matériaux ont été comparés : une céramique polycristalline frittée, des céramiques texturées spéciales et des bicristaux obtenus par la méthode des flux.

Les céramiques ont été élaborées au CRISMAT (Caen) par Desgardin et al. En ce qui concerne le matériau polycristallin de référence, la poudre a subi un pressage isostatique et un frittage de $30 \mathrm{~h}$ à $950^{\circ} \mathrm{C}$. La montée et la descente en température ont été effectuées, sous flux d’oxygène, à la vitesse de $50^{\circ} \mathrm{C} / \mathrm{h}$. Le matériau est homogène et constitué essentiellement de grains de la phase «123» dont la taille est comprise entre 2 et $10 \mu \mathrm{m}$. Les échantillons ont ensuite été polis et amincis ioniquement pour l'observation en microscopie électronique en transmission.

Le deuxième type de céramique est obtenu dans les conditions thermiques habituelles de la méthode M.T.G. [3]. En complément, la forme spécifique de l'échantillon (en W) induit de gros grains de l'ordre du $\mathrm{mm}$ et des joints de forte désorientation, desquelles seront prélevées des «éprouvettes " contiguës pour la microscopie électronique et les mesures électriques.

Les bicristaux sont extraits de grappes de monocristaux préparés par la méthode des flux, au laboratoire de Chimie Minérale de l'Université de Rennes I par H. Noel.

Cristallographie et STRUCtURE ATOMiQue InTERFACIAle. - Les images de structure interfaciale (HREM) ont été obtenues à $400 \mathrm{kV}$ sur le microscope électronique Jeol $4000 \mathrm{EX}$ du CENG Grenoble avec une résolution spatiale de $0,17 \mathrm{~nm}$ et un porte-objet double inclinaison $\left( \pm 20^{\circ}\right)$. Les relations d'orientation entre grains et l'indice de coïncidence du bicristal $[4,5]$ ont été déterminées par analyse des diagrammes de Kikuchi [6].

COMPORTEMENT ÉLECTRIQUE DES JOINTS DE GRAINS. - Le comportement électrique des joints a été étudié sur des bicristaux, élaborés par la méthode des flux ou prélevés sur les céramiques texturées spéciales. Les microcontacts sont réalisés par évaporation d'argent au travers de masques adaptés à la géométrie des cristaux. Toutefois, quand le faciès des bicristaux est trop compliqué ces contacts sont tracés au pinceau avec la laque d'argent. Les mesures de courant critique sont effectuées sous microscope optique, à l'aide de microélectrodes que l'on place successivement sur les 2 contacts intra ou intergranulaires dans un cryostat refroidi à l'azote liquide $(71 \mathrm{~K})[1]$.

\section{Description des joints de grains.}

L'observation d'une céramique polycristalline frittée montre une forte proportion de joints contenant le plan $(a b)$. Nous classerons les joints en 2 groupes, suivant qu'ils contiennent ou 
non ce plan. Nous verrons que cette classification est univoque par rapport au comportement électrique des joints.

JoINTS CONTENANT LE PLAN $(a b)$. Ils présentent essentiellement deux cas de figures. S'il n'existe pas de relation d'orientation entre les grains adjacents, le joint présente une forte énergie qui est généralement minimisée par la présence d'un film non cristallin intergranulaire, résidu de la phase liquide (Fig. 1). Par contre, s'il existe une relation d'orientation entre les grains, le joint présentera une énergie beaucoup plus faible, qui se traduit par l'absence systématique de phase intergranulaire. De plus, à l'échelle atomique, on constate que ces joints présentent une structure atomique interfaciale continue. Le plus bel exemple est représenté par le joint de coïncidence $\Sigma_{\mathrm{Q}} 3$ (Fig. 2) où les axes $c_{1}$ et $c_{2}$ des grains adjacents sont orthogonaux et la maille de coïncidence est triple de la maille de base (approximation de la maille orthorhombique par une maille quadratique $\mathrm{Q}$ de paramètre $c=3 a$ ).

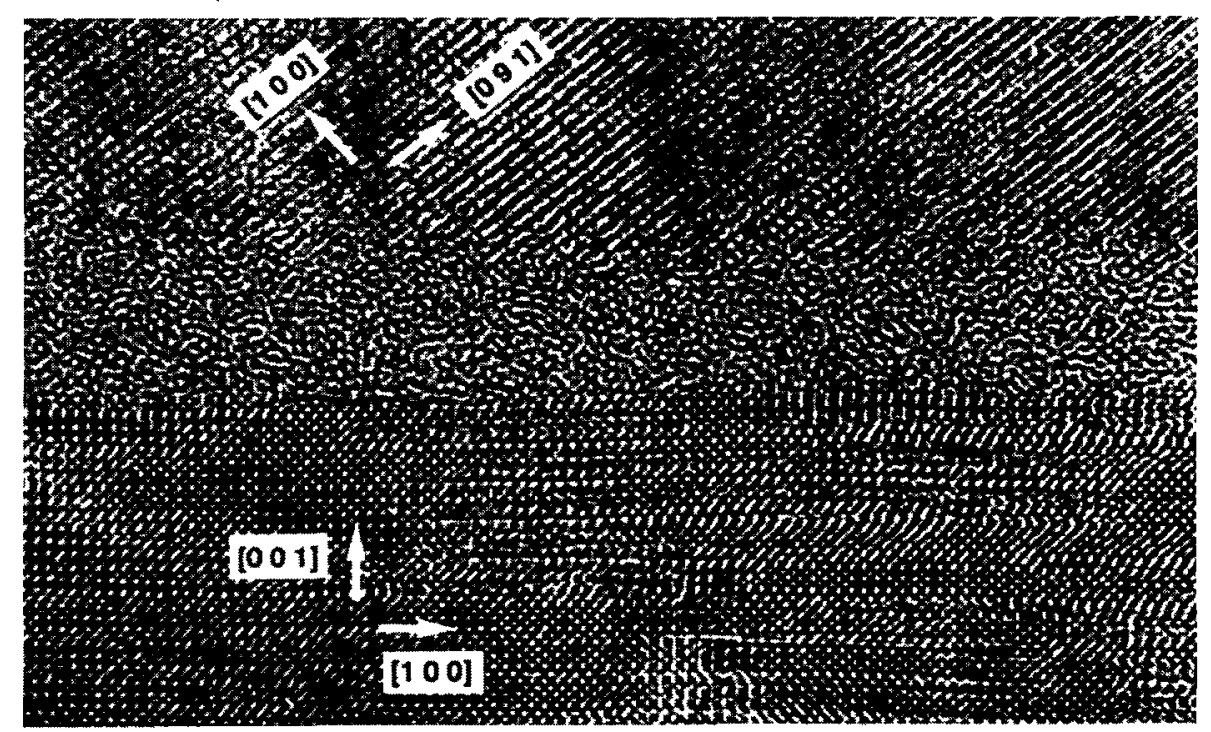

Fig. 1. - Film intergranulaire non cristallin sur un joint général contenant le plan $(a b)$ : image de structure, $400 \mathrm{kV}$ (HREM).

[Non-crystalline intergranular film on a general boundary containing the $(a, b)$ plane : structure image, $400 \mathrm{kV}($ HREM).]

Par ailleurs, nous considérons un cas intermédiaire, correspondant à la figure 3 , qui s'avère particulièrement intéressant du point de vue structural. Il s'agit d'un joint mixte correspondant à une flexion de $5^{\circ}$ autour de [100] et à une torsion de $27^{\circ}$ autour de [001]. La légère désorientation entre les axes $c$ entraîne une géométrie très simple du joint, qui se traduit par un facettage utilisant au maximum le plan basal (001) sur chaque grain. Il en résulte un maillage constitué de zones alternées de bon cristal $(\mathrm{BC})$ doté d'une structure atomique quasi-parfaite et de zones d'accommodation (A) où le réseau est très perturbé. Les zones de bon cristal s'étendent sur $\cong 8 \mathrm{~nm}$ de large. On notera sur le grain du bas de cette figure de même que sur celui de la figure 1 , des défauts d'empilement parallèles au plan $(a, b)$ de type « 124 assez fréquents. Ces défauts sont distribués sur l'ensemble du grain et ne sont pas localisés aux 


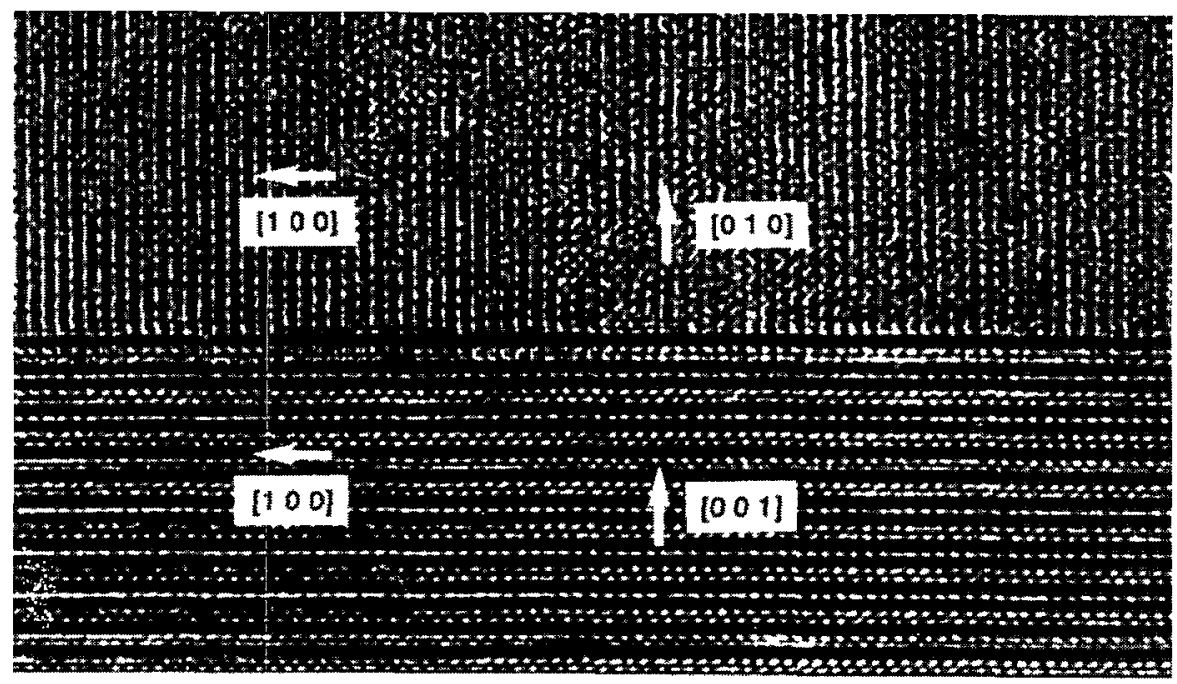

Fig. 2. - Joint $\Sigma_{Q} 3\left(c_{1} \perp c_{2}\right)$ contenant le plan $(a b)$.

[ $\Sigma_{Q} 3$ boundary $\left(c_{1} \perp c_{2}\right)$ containing the $(a, b)$ plane.]

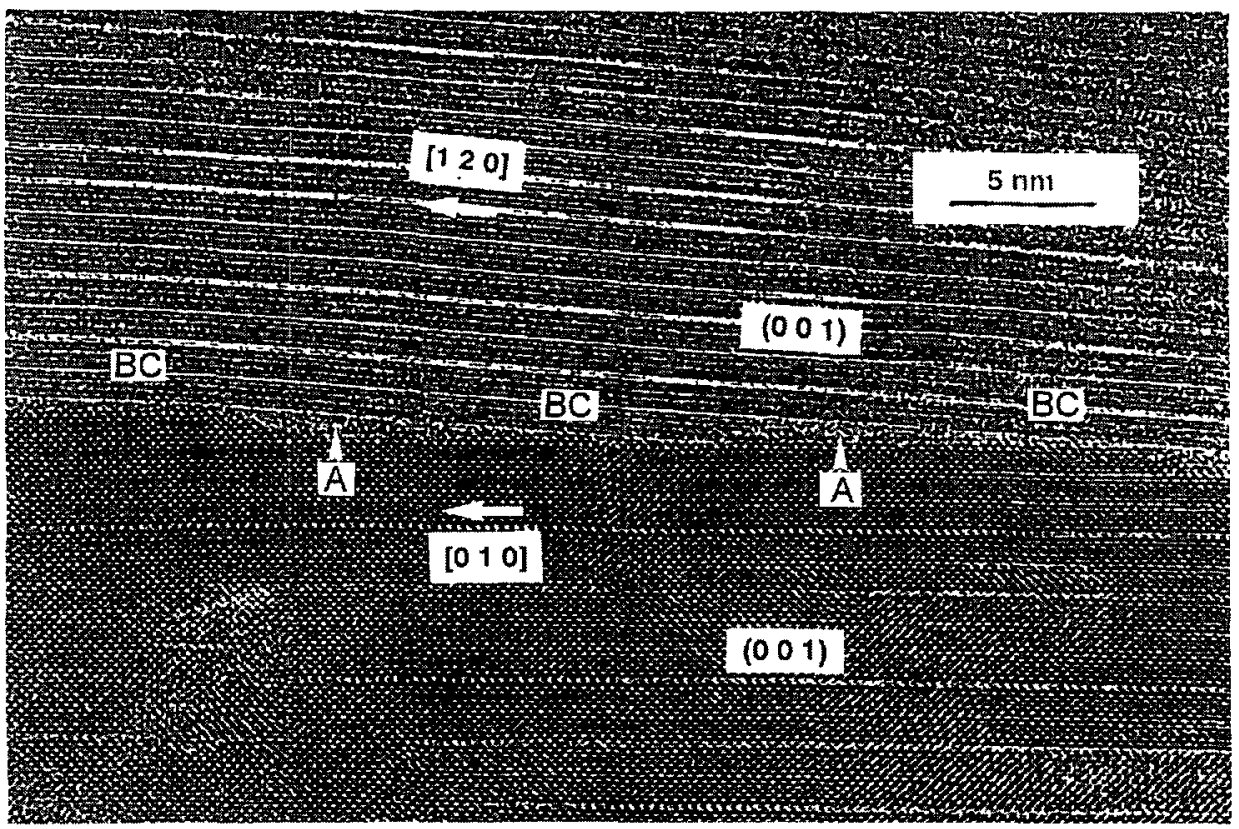

Fig. 3. - HREM : joint mixte contenant le plan $(a b) \theta_{\text {flevon }}=5^{\circ} /[100] \theta_{\text {tor } 10 n}=27^{\circ} /[001]$.

[HREM : mixed boundary containing the $(a, b)$ plane : $\theta_{\mathrm{tllt}}=5^{\circ} /[100] \theta_{\mathrm{tust}}=27^{\circ} /[001]$.] 
interfaces. Par ailleurs leur longueur est relativement réduite par rapport à celle du joint. Ils n`influenceront donc que faiblement les mesures électriques locales intra ou intergranulaires.

JOINTS ADJACENTS. - Nous classons sous ce vocable les joints ne contenant pas le plan (ab). Ils présentent en général, une géométrie très compliquée liée à la croissance cristalline préférentielle dans ce plan. Leur observation en microscopie électronique haute résolution s'avère par conséquent extrêmement délicate. La figure 4 montre un exemple, tout à fait caractéristique, de ce type de joint. On voit tout d'abord, que le joint ne contient pas, généralement, l'axe $c$. De plus, le joint correspond à des plans très compliqués (ici (156) et (423)). Il est remarquable. en dépit de l'absence de relation de coïncidence, que le désordre structural interfacial ne s'étende pas sur plus de $0,5 \mathrm{~nm}$ de part et d'autre du joint. Ceci est très spécifique des joints ne contenant pas le plan $(a b)$.

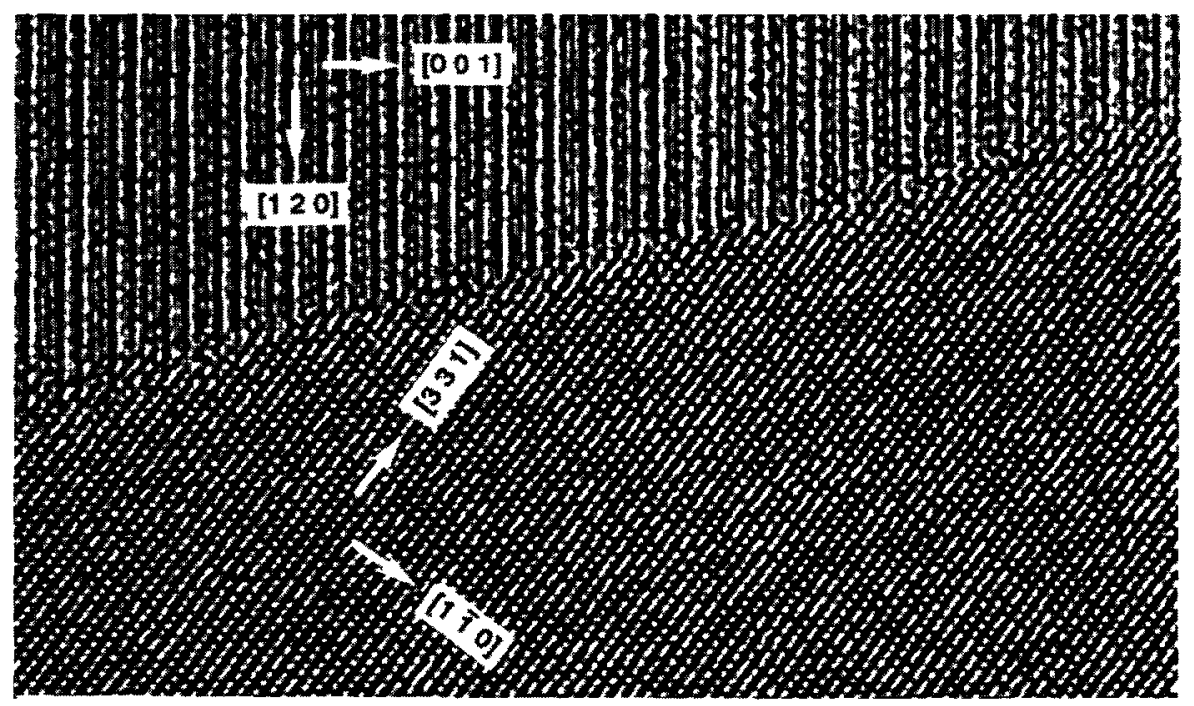

Fig. 4. - HREM : joint adjacent ne contenant pas le plan $(a b)$ et ne présentant aucune relation simple de coincidence.

[HREM : adjacent boundary not containing the $(a, b)$ plane and which does not exhibit any simple coincidence relationshp.]

$\mathrm{Si}$, de plus, ces joints présentent une relation de coüncidence, le désordre atomique interfacial est minimisé. Le cas le plus remarquable est celui de la figure 5 qui montre un pur joint de flexion $\left(\theta=43^{\circ}\right)$ autour de l'axe c. correspondant à l'indice de coïncidence $\Sigma 29$. La flexion proche de $45^{\circ}$ permet au joint de se facetter d'une façon particulièrement simple suivant la séquence

$$
(010)_{1} / /(110)_{2}-(110)_{1} / /(010)_{2} \cdot
$$

Cette géométrie remarquable confère au joint une très faible énergie qui est révélée par l'absence de contraste localisé au niveau de l'interface et par une structure atomique quasiparfaite. Une désorientation de $45^{\circ}$ entre les grains a été fréquemment observée sur les couches minces $[7,8]$. 


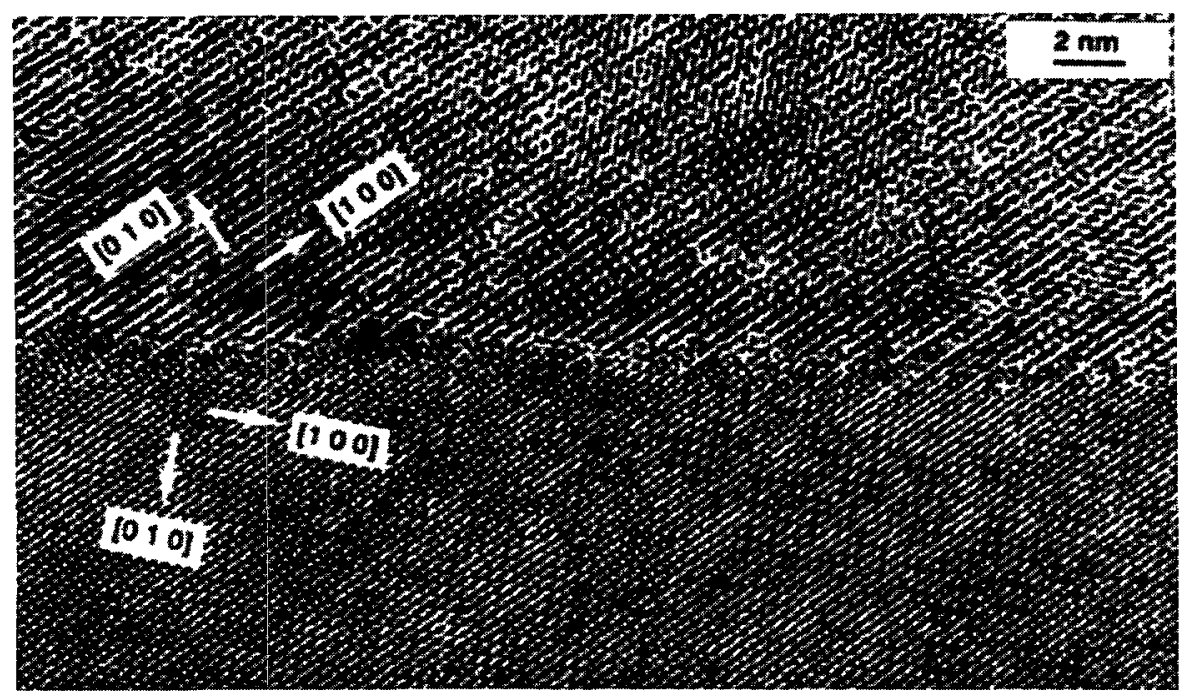

Fig. 5. - HREM : joint $\Sigma 29$ facetté suivant la séquence $(010)_{1} / /(110)_{2}-(110)_{1} / /(010)_{2}$.

[HREM : $\Sigma 29$ boundary faceted according to the sequence $(010)_{1} / /(110)_{2}-(110)_{1} / /(010)_{2}$.]

Comportement électrique des joints de grains.

Etant donnée la relation directe établie entre la cristallographie et la structure atomique des joints, il semblait particulièrement approprié de comparer le comportement électrique des deux groupes de joints précédemment répertoriés.

Pour tester les joints généraux contenant le plan $(a b)$, nous avons utilisé les bicristaux, obtenus par la méthode des flux, qui se caractérisent par des joints de forte énergie, le plus souvent dotés d'une seconde phase intergranulaire. La figure 6 montre les courbes

$\approx$

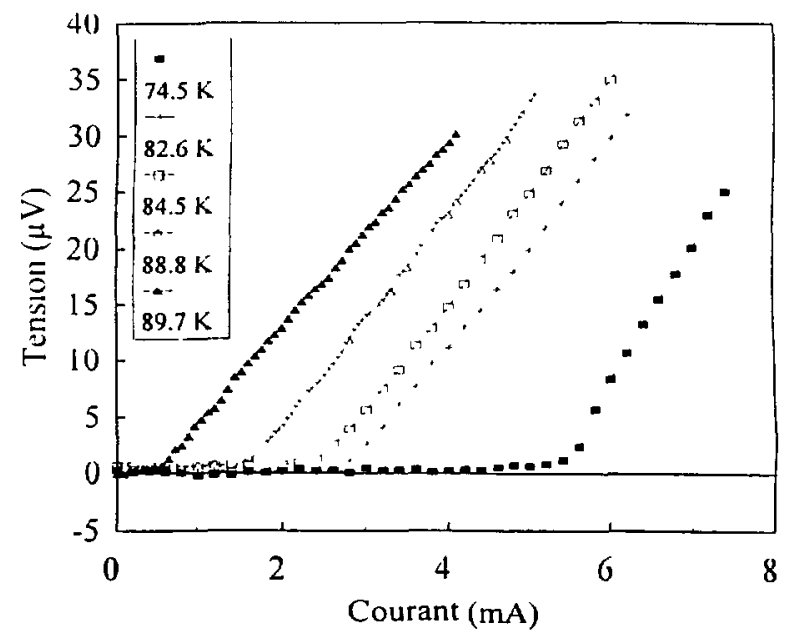

Fig. 6. - Caractéristiques courant-tension sur un joint général de forte énergie, contenant le plan $(a b)$.

[Current-voltage characteristics on a high-energy general boundary containing the $(a, b)$ plane.] 
$I(V)$, correspondant à des températures comprises entre 74 et $90 \mathrm{~K}$, caractéristiques de ce type de joint. Tous les échantillons donnent de très faibles $J_{\mathrm{c}}$, qui se situent dans la gamme de l'Ampère $/ \mathrm{cm}^{2}$ à $74 \mathrm{~K}$, alors que le courant critique intragranulaire mesuré par aimantation, dans les cristaux est de $3 \times 10^{3} \mathrm{~A} / \mathrm{cm}^{2}$ à $70 \mathrm{~K}$. Il apparaît donc un coefficient d'atténuation de ce courant tout à fait rédhibitoire.

La deuxième série de mesures locales a porté sur les joints de coïncidence ne contenant pas le plan $(a b)$, spécialement élaborés sous gradient thermique en fonction de la forme de l'échantillon. La figure 7a montre l'image haute résolution d'un des joints étudiés, qui correspond à un indice de coïncidence $\Sigma 7$. Bien que la géométrie du plan des joints soit compliquée, on voit que le désordre atomique interfacial ne dépasse pas $0,5 \mathrm{~nm}$ d'épaisseur.

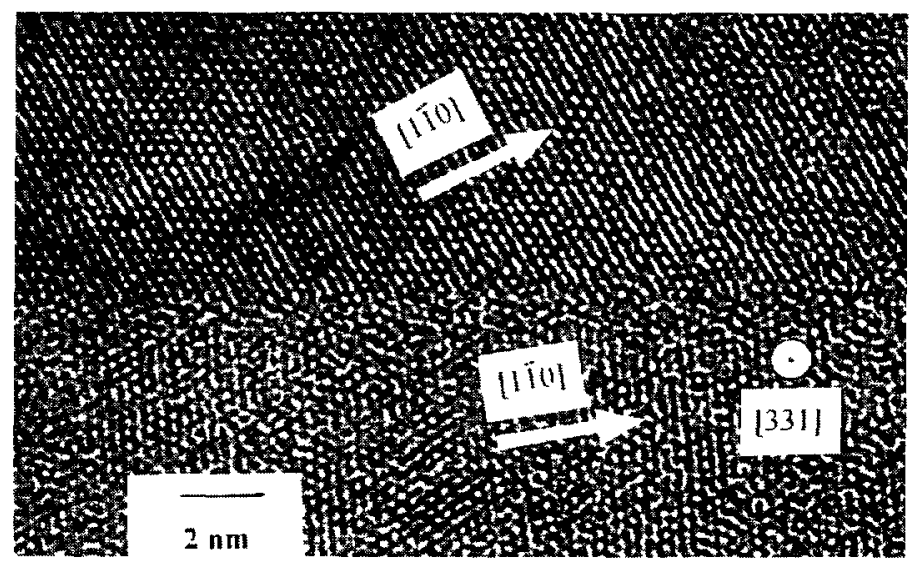

a)

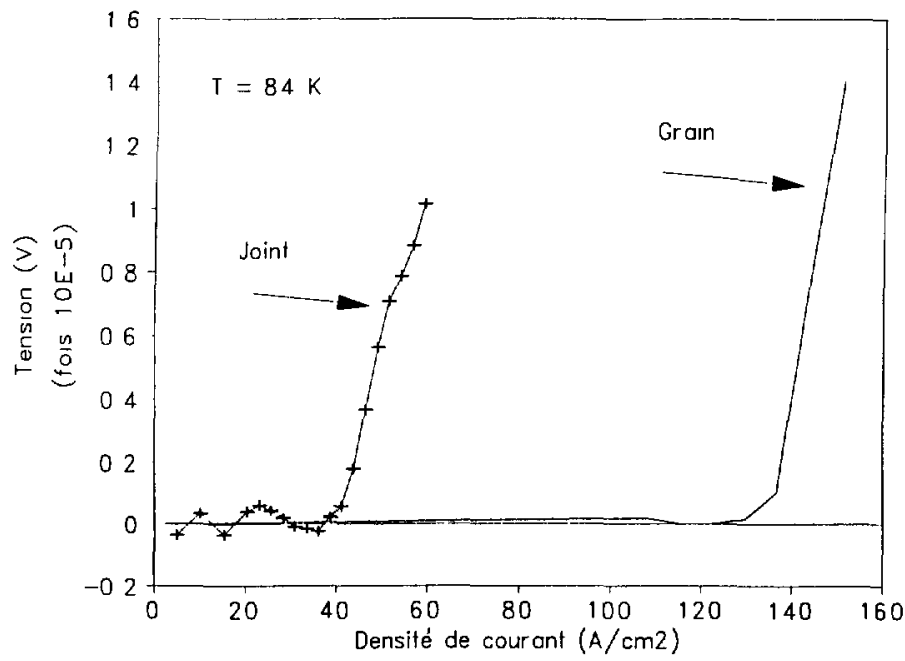

b)

Fig. 7. - Joint de coincidence $\Sigma 7$ ne contenant pas le plan $(a b)$ a) Image de structure. b) Caractéristiques courant-tension sur le joint et sur le grain adjacent.

[ $\Sigma 7$ coincidence boundary not containing the $(a, b)$ plane: a) structure image, b) current-voltage characteristics on the boundary and on the adjacent grain.] 
La figure $7 \mathrm{~b}$ indique les caractéristiques courant-tension obtenues sur ce joint et sur le grain adjacent. Les courants critiques respectifs sont de 40 et $130 \mathrm{~A} / \mathrm{cm}^{2}$ à $84 \mathrm{~K}$, soit un coefficient d'atténuation de l'ordre de 3. Les mesures effectuées sur différents types de joints de coïncidence obtenus par cette méthode indiquent toujours des valeurs de ce coefficient comprises entre 2,5 et 5 .

\section{Discussion.}

La comparaison entre les propriétés électriques et structurales des différents types de joints permet d'établir une relation directe entre l'atténuation du courant critique par ces joints et la structure atomique interfaciale.

Il apparaît que les joints contenant le plan $(a b)$ donneront presque toujours des atténuations extrêmement fortes $(>100)$. Le comportement électrique de ces joints s explique bien à partir des observations structurales effectuées en TEM. En effet, en ce qui concerne la transmission du courant supraconducteur. on peut distinguer essentiellement trois types de microstructures interfaciales contenant le plan $(a b)$ : les interfaces planes avec ou sans phase intergranulaire et les interfaces facettées. Les joints plans atténueront toujours fortement le courant critique en raison de leur géométrie défavorable (l'axe $c$ est perpendiculaire au plan du joint pour au moins un des deux grains). Par ailleurs les joints facettés de ce type seront également contre-indiqués, puisque nous avons montré [1] que les facettes non parallèles à $(a b)$ qui pourraient permettre le passage du supracourant sont toujours bordées par une seconde phase non cristalline de 2 à $3 \mathrm{~nm}$ d'épaisseur, qui atténue très fortement ce courant.

Toutefois, en attendant d'obtenir des résultats directs sur le comportement électrique des joints du type de celui de la figure 3. on peut supposer que ces joints intermédiaires sont susceptibles de participer au passage du courant supraconducteur, au moyen du maillage de «bon cristal» qui les caractérisent.

Par contre, les joints ne contenant pas le plan $(a b)$ et tels que les grains adjacents sont en relation de coïncidence, se distinguent toujours par une faible atténuation du courant critique $(<5)$. Cela s'explique tout d'abord par une géométrie favorable par rapport à la direction d'anisotropie c et ensuite par une optimisation de la structure atomique interfaciale liée à l'existence d'un réseau de coïncidence des sites réticulaires, dans le plan du joint, qui entraînera, dans les cas les plus favorables, des coïncidences atomiques pour les deux grains et de toute façon, une minimisation des déplacements atomiques au niveau de l'interface, et, par conséquent, une dépression réduite du paramètre d'ordre. Il serait, en outre, particulièrement intéressant de tester le comportement électrique des joints $\Sigma 29$ qui devraient induire une très faible atténuation du courant critique $(<3)$. étant donnés les résultats obtenus pour les coïncidences $\Sigma 7$ et $\Sigma \mathbf{2 5}$ qui correspondent à des joints dont la cristallographie est nettement moins favorable (plans moins denses ne contenant pas l'axe c). De plus, signalons que le facettage, tel que celui observé sur le $\Sigma 29$, c est-à-dire comprenant des microfacettes pseudopériodiques de quelques $\mathrm{nm}$ de long, fréquent sur ces différents joints, est extrêmement favorable pour le piégeage des vortex.

\section{Conclusion.}

Nous avons comparé la structure atomique et le comportement électrique des différents types de joints dans les céramiques frittées $\mathrm{YBa}_{2} \mathrm{Cu}_{3} \mathrm{O}_{7-1}$, au moyen de la microscopie électronique haute résolution et d'une microsonde électrique basse température, développée au laboratoire, et qui permet de mesurer très localement le courant critique, tout en visualisant la zone sous microscope optique.

Il apparaît que ces propriétés sont étroitement corrélées et qu elles varient très largement en fonction de la cristallographie du joint. 
Les joints de grains contenant le plan $(a b)$ se caractérisent, sauf exception, par une forte atténuation du courant critique. Ils serviront en général comme « rampe de circulation » pour les paires de Cooper.

Par contre les joints adjacents ne contenant pas le plan $(a b)$ seront susceptibles de ne provoquer qu une faible atténuation $(<5)$ du courant supraconducteur dès l'instant où ils bordent des grains en relation de coïncidence.

Si l'on remonte à l'échelle macroscopique et si l'on suppose, à titre estimatif, que $5 \%$ des joints contenant le plan $(a b)$ sont capables d'assurer un passage suffisant du courant supraconducteur ; il faudrait pour obtenir un courant critique satisfaisant que $10 \%$ des joints soient de coïncidence et ne contiennent pas le plan $(a b)$.

L'obtention d'un courant critique élevé $\left(\geqslant 10^{4} \mathrm{~A} / \mathrm{cm}^{2}\right)$ sur les céramiques polycristallines implique donc, de définir les conditions de frittage susceptibles d'optimiser la proportion de ces joints spéciaux.

\section{Remerciements.}

Nous tenons à remercier Isabelle Monot (Crismat Caen) pour le soin et la patience dont elle a fait preuve pour l'élaboration des échantillons céramiques. H. Noel (Université de Rennes I) pour les nombreux bicristaux fournis et $J$. Dessus (CEA-Grenoble) pour son amicale assistance sur le microscope 400EX.

\section{Bibliographie}

11] Laval J. Y., Drouet M. et Delamarre C., Structure et propriétés électriques de joints de grans favorables au passage du courant supraconducteur dans les céramiques à haute $T_{\llcorner}, I$. Phis III France 1 (1991) 1795-1803.

[2] Laval J. Y., Drouet M., Swiatnich1 W., Cabanel C., Delamarre C., Monot I. and Desgardin G., Structure and electrical behaviour of grain boundaries allowing large critical currents in ceramics superconductors, Mater Scl Folum 126-128 (1993) 733-736.

[3] Wang J., Tessler L., Desgardin G., Maignan A., Provost J. et Raveau B.. Synthèse et caractérısatıon de céramıques YBaCuO fortement texturées, I Phys III France 2 (1992) 225-231.

[4] Grımmer H.. Bollmann W. and Warrıngton D. H.. Coincidence-site lattices and complete pattemsshift lattices in cubic crystals, Acta Civst. A 30 (1974) 197.

[5] Singh A., Chandrasekhar N. and Kıng A. H . Comcidence orientations of crystals in tetragonal systems with applications to $\mathrm{YBa}_{2} \mathrm{Cu}_{3} \mathrm{O}_{7-n}$, Acta $C_{1}$ yst. B 46 (1990) 117.

|6| Pumphrey P. H. and Bowkett K. M., An accurate method for determining crystallographıc orientation by electron diffraction. Phys. Status Solıd A 2 (1970) 339.

17] McKernan S., Norton M. G. and Carter C. B., The $45^{\circ}$ grain boundaries in $\mathrm{YBa}_{3} \mathrm{Cu}_{3} \mathrm{O}_{7-}$, I Mater. Res 7 (1992) 1052.

[8] Ramesh R., Hwang D., Ravı T. S., Inam A., Barner J. B., Nazar L., Chan S. W., Chen C. Y., Dutta B., Venkatesan T. and Wu X. D.. Epitaxy of Y-BaCu-O thin films grown on singlecrystal MgO, Appl Phvs Lett 56 (1990) 2243. 